\title{
Valorando bienes y servicios ambientales (BSA) del manglar en la ostricultura artesanal: Las Tunas, Cuba
}

\section{Valuing environmental goods and services (EGS) of the mangrove in artisanal oyster farming: Las Tunas, Cuba}

\begin{abstract}
Abel Betanzos-Vega 1 [D
Arturo Tripp-Quezada 2 (D)

Esnoldo Macías-Aguilera ${ }^{3}$

Adalberto Leyva-Segura ${ }^{4}$,

Gustavo Arencibia-Carballo 1 (1)

José Manuel Mazón-Suástegui

${ }^{1}$ Centro de Investigaciones Pesqueras

(CIP), Calle 246 y 5ta Ave, CP. 19100.

Playa, La Habana, Cuba.

${ }^{2}$ Instituto Politécnico Nacional, Unidad

Centro Interdisciplinario de Ciencias

Marinas (CICIMAR), Col. Playa Palo

Santa Rita, CP. 23096. La Paz,

México.

${ }^{3}$ Unidad Empresarial Básica "Juanito

Mora", calle Baire 42, Puerto Padre,

Las Tunas, Cuba.

${ }^{4}$ Empresa Pesquera de Las Tunas

(PESCATUN), calle $65 \# \cdot 2$. Esq.

Circunvalación Sur, La Loma, CP.

75100 , Las Tunas, Cuba.

${ }^{5}$ Centro de Investigaciones Biológicas

del Noroeste, S.C. (CIBNOR). Calle

I.P.N. 195, Col. Playa Palo de Santa

Rita Sur, CP. 23096. La Paz, B.C.S.,

México.
\end{abstract}

*Autor de correspondencia:

jmazon04@cibnor.mx

Artículo científico

Recibido: 30 de enero 2021

Aceptado: 17 de enero 2022

Como citar: Betanzos-Vega A Tripp-Quezada A, Macías-Aguilera E, Leyva-Segura A, Arencibia-Carballo G, Mazón-Suástegui JM (2022) Valorando bienes y servicios ambientales (BSA) del manglar en la ostricultura artesanal: Las Tunas, Cuba. Ecosistemas y Recursos Agropecuarios 9(1): e2829. DOI: 10.19136/era.a9n1.2829
RESUMEN. En Cuba, la producción de ostión de mangle Crassostrea rhizophorae proviene de la pesca extractiva $(75 \%)$ y del cultivo artesanal $(25 \%)$. En la pesca extractiva ocurre descortezado o corte de raíces de mangle rojo (Rhizophora mangle) durante la recolecta de ostión. En el cultivo se utilizan troncos y ramas de mangle para las granjas y confección de colectores, lo que daña al ecosistema y reduce la oferta de bienes y servicios ambientales (BSA) que presta el manglar. Con el objetivo de evaluar mediante un análisis de costo-beneficio la actividad ostrícola en Puerto Padre, Las Tunas, Cuba, y determinar la viabilidad económica y ambiental de la ostricultura artesanal, se aplicaron estrategias productivas con enfoque ecosistémico incluyendo un valor ecológico representativo del beneficio bruto de las funciones del manglar. El cultivo artesanal realizado es una alternativa de producción económica y ambientalmente viable, con mayor rendimiento promedio en carne de ostión (6.4\%), versus rendimiento en carne de la pesca extractiva (5.8\%). La inclusión, en el análisis de costo-beneficio de la actividad ostrícola, del costo por daños y beneficios al manglar, indica la necesidad de incluir la valoración de BSA en los mecanismos tradicionales de evaluación económica.

Palabras clave: Beneficio ambiental, ostión de mangle, valor ecológico.

ABSTRACT. In Cuba, the production of mangrove oyster Crassostrea rhizophorae comes from extractive fishing $(75 \%)$ and artisanal cultivation (25\%). In extractive fishing, the barking or cutting of the roots of the red mangrove (Rhizophora mangle) occurs during the collection of oysters. In cultivation, mangrove trunks and branches are used for farms and for making collectors, which damages the ecosystem and reduces the supply of environmental goods and services (BSA) provided by the mangrove. With the objective of evaluating through a cost-benefit analysis the oyster farming activity in Puerto Padre, Las Tunas, Cuba, and determining the economic and environmental viability of artisanal oyster farming, productive strategies with an ecosystemic approach were applied, including an ecological value representative of the benefit gross of mangrove functions. Artisanal farming turned out to be an economically and environmentally viable production alternative, which allowed a higher average yield in oyster meat $(6.4 \%)$, versus meat yield from extractive fishing $(5.8 \%)$. The inclusion, in the cost-benefit analysis of the oyster farming activity, of the cost for damages and benefits to the mangrove swamp, indicates the need to include the BSA valuation in the traditional mechanisms of economic evaluation.

Key words: Environmental benefit, mangrove oyster, ecological value. 


\section{INTRODUCCIÓN}

El manglar es uno de los ecosistemas de mayor aporte a la biodiversidad y abundancia de especies marinas en Cuba, al brindar servicios ecosistémicos que garantizan importantes actividades socioeconómicas (Alcolado et al. 2007, Gómez-País 2007). Diversas actividades humanas, incluyendo la pesca, hacen uso directo e indirecto de los bienes y servicios del manglar (BSA), mayormente como fuente de abastecimiento de materias primas, y los usuarios no siempre entienden la responsabilidad de su valoración y protección, no solo por su consecuencia en la biodiversidad, sino además por su impacto socioeconómico (Palacios y Cantera 2017, López 2019).

La información acerca de alternativas productivas y canales de mercado para la producción eco-amigable es escasa, y no existe una tradición de otorgar un valor económico a los BSA, ni mecanismos para incorporarlos al sistema de evaluación económica; es decir, en los análisis económicos de costo-beneficio de las actividades productivas (Barsev 2008). Lo anterior justifica la propuesta de nuevos mecanismos financieros que permitan otorgar un valor económico a los BSA, como estrategia de conservación y uso sostenible de la biodiversidad (Gómez-País 2007).

En Cuba, el ostión de mangle (OM) Crassostrea rhizophorae (Guilding, 1828) contribuye con el $80 \%$ de la producción ostrícola nacional ( 800 a 1000 t anuales), y el 75\% de esa producción proviene de la actividad extractiva pesquera (Mazón-Suástegui et al. 2019). Esta actividad implica el descortezado o el corte de raíces y ramas de mangle rojo (Rhizophora mangle Linnaeus 1753) para acceder al ostión; y es tradición en Cuba el uso de troncos y ramas de mangle para construir las empalizadas de las granjas ostrícolas y en manufactura de colectores de semilla silvestre (Betanzos-Vega et al. 2014).

Después de una intensa actividad pesquera entre 1990 y 2000 en la bahía de Puerto Padre, Cuba, entidades conservacionistas del ministerio de ciencia, tecnología y medio ambiente (CITMA) y la autoridad de regulación pesquera nacional, aprobaron de manera precautoria un acuerdo de moratoria de pesca, que prohíbe la pesca comercial de peces y de otros recursos marinos al interior de esta bahía (Betanzos-Vega et al. 2020). En este contexto, sólo la actividad ostrícola de OM se permite como acción de pesca comercial, condicionada a ser eco-amigable con el manglar (Betanzos-Vega et al. 2020). El desarrollo y extensión de la ostricultura en la bahía de Puerto Padre es necesaria debido a su importancia como fuente de empleo e ingresos para la región, pero se requiere demostrar que dicha actividad es compatible con la preservación de BSA del manglar. El objetivo de este trabajo fue evaluar la viabilidad económica y ambiental de un proyecto de ostricultura artesanal ecológicamente amigable, con un análisis de costo-beneficio, teniendo en cuenta el efecto de la actividad ostrícola en los BSA del manglar y su interrelación.

\section{MATERIALES Y MÉTODOS}

\section{Descripción del área de estudio}

La bahía de Puerto Padre se localiza al norte de la provincia de Las Tunas, en la región nororiental de Cuba, con punto de acceso en los $21^{\circ} 16^{\prime} 30.5^{\prime \prime}$ LN y $076^{\circ} 31^{\prime} 49.8^{\prime \prime}$ LO. La bahía se divide en dos grandes lóbulos, occidental y oriental, en el occidental se ubica la ciudad de Puerto Padre y el puerto Carúpano (Figura 1). El lóbulo oriental, de menor urbanización, presenta zonas bajas y humedales al noreste con presencia mayoritaria de mangle rojo ( $R$. mangle), donde se localizan los bancos naturales de OM. El clima de la región es húmedo y durante el verano se alcanzan temperaturas de 30 a $34{ }^{\circ} \mathrm{C}$, con temperatura media anual del aire de 24 a $26{ }^{\circ} \mathrm{C}$, y acumulado anual de lluvia entre 800 y $1200 \mathrm{~mm}$ (ACC 1989).

\section{Colecta y procesamiento de datos}

El ostión en Cuba se comercializa desconchado, como masa o carne de ostión en salmuera, mayormente en bolsas de nailon de $1 \mathrm{~kg}$ (BetanzosVega et al. 2018). De la estadística (2010 2019) del establecimiento pesquero de Puerto Padre, y de la empresa pesquera provincial PESCATUN, 


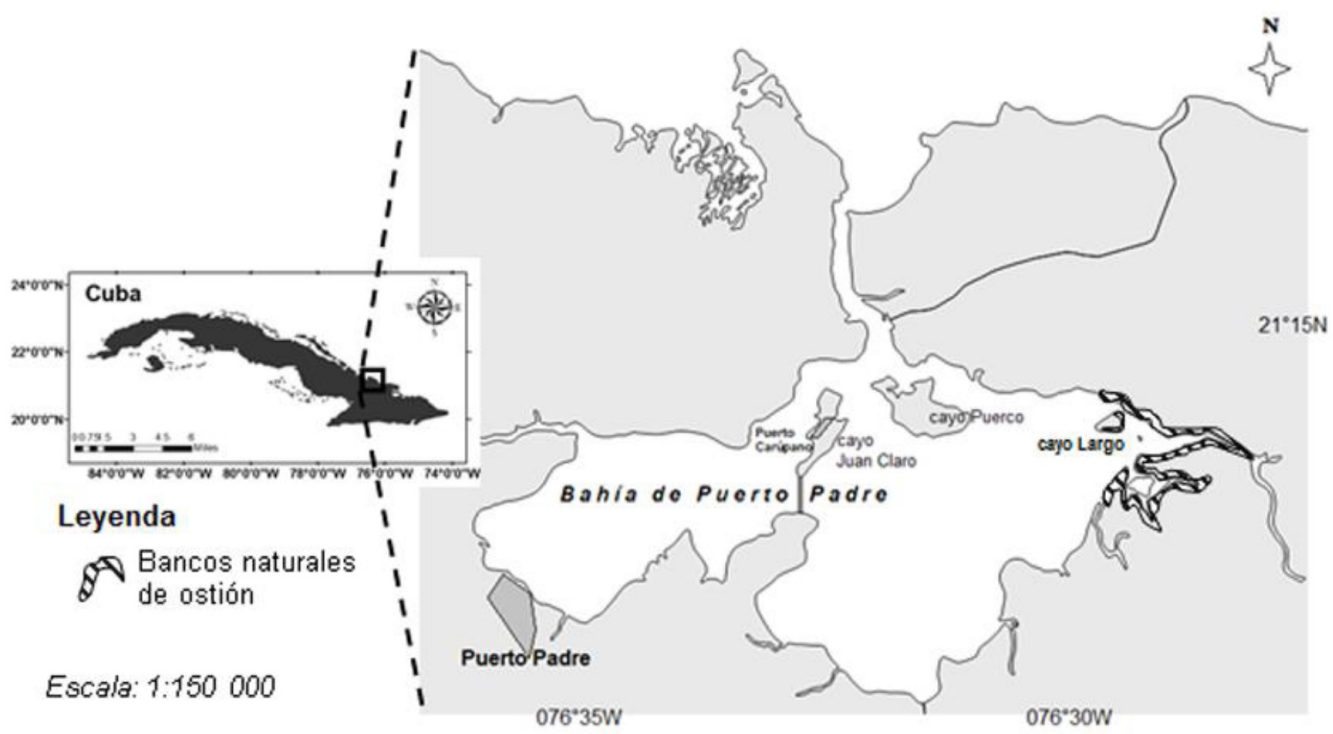

Figura 1. Área de estudio, bahía de Puerto Padre, Las Tunas, Cuba. Al noreste los bancos de ostión, y en cayo Largo se ubica la granja de cultivo de ostión.

se obtuvieron datos anuales de producción de ostión, y del periodo 2015 - 2019 datos de producción (pesca y cultivo) en toneladas de ostión en concha y de su rendimiento en carne (\%) para fines de comercialización. El rendimiento en carne $(\mathrm{R})$ se obtiene aplicando la formula $R=$ peso de la carne de ostión obtenida / peso del ostión en su concha x 100 (Rebelo et al. 2005, Betanzos-Vega et al. 2018), y se utiliza en Cuba como indicador de productividad, siendo aceptable un valor mayor igual al 4.9\% (Mazón-Suástegui et al. 2019). También se obtuvieron datos operativos y productivos de una granja ostrícola en operación del 2015 al 2019. Durante 2015 y 2016, se instalaron 7000 y 5000 colectores de ostión respectivamente, todos confeccionados con ramas de mangle. En 2017 la cantidad se incrementó a 16000 , y durante 2018 y 2019 se instalaron más de 30000 colectores. Como estrategia de ostricultura eco-amigable, en 2019 se probaron dos tipos de colector para determinar la productividad y potencialidad para sustituir el uso del colector de ramas de mangle. En unidades de cultivo para fijación de ostión se intercalaron (mayo de 2019) colectores de conchas de ostión (200 colectores) y de alambre de aluminio (100 colectores) entre los colectores tradicionales de ramas de mangle, después de fijación natural de semillas, se colocaron aleatoriamente en la granja de engorde. En cosecha (noviembre de 2019), se tomó una muestra no selectiva de 30 colectores de cada tipo, y de cada colector se obtuvieron y pesaron todos los ostiones vivos, para determinar rendimiento en peso en kilogramos $(\mathrm{kg})$, con una balanza analógica con rango de 0 a $5 \mathrm{~kg}$ y precisión de $10 \mathrm{~g}$, marca Polder, modelo 985-75, USA, por tipo de colector utilizado: ramas de mangle (Figura $2 \mathrm{~A}$ ), alambre de aluminio (Figura 2B), y sartas de concha de ostión (Figura 2C).

Con el programa Maplnfo versión 8, se estimó un área (850 ha) de manglares al noreste de la bahía (Figura 1), y con apoyo de un GPS (Garmin 48) en el terreno (noviembre de 2019), se determinó el área (1.3 ha) de una franja de línea de costa de $5.86 \mathrm{~km}$ de mangle rojo ( $R$. mangle), con anchura promedio de $1.2 \mathrm{~m}$ desde la pared del manglar (entramado de raíces) hasta el extremo exterior de las ramas de mangle, donde se distribuyen los ostiones silvestres. Para obtener los datos relativos de la afectación a los BSA del ecosistema de manglar, se estimó un área de impacto por pesca extractiva de ostión silvestre de 0.70 ha de mangle, en sitios donde se ha realizado descortezado y corte de raíces, para acceder al ostión. Según el criterio de los pescadores ostioneros 


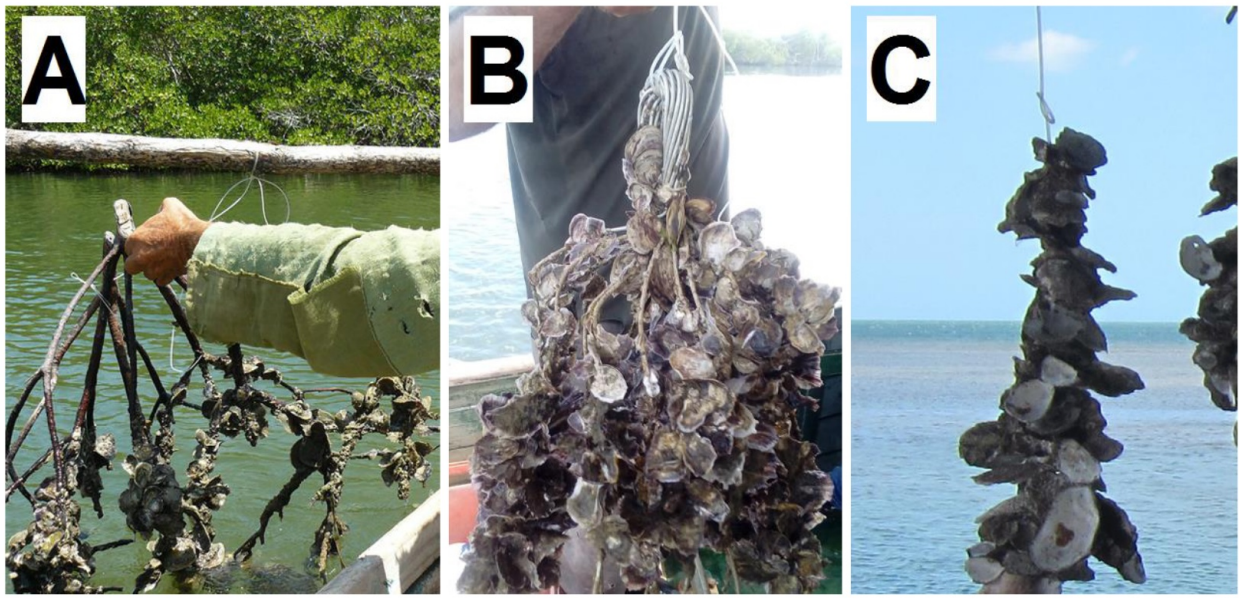

Figura 2. Colectores utilizados para la captación de larvas y semillas de ostión de mangle Crassostrea rhizophorae: Ramas de mangle (A), alambre de aluminio (B) y concha madre de ostión (C).

que operan en la zona, entre 2015 y parte de 2018 , en un área de aproximadamente 3.3 ha, por detrás de la línea de costa al interior del bosque de manglar, se ha realizado tala y corte de ramas de mangles para la obtención de troncos y varas para la empalizada de granjas ostrícolas, y corte de ramas terminales del mangle rojo para la confección anual de colectores. Estos datos y los resultantes de la evaluación in situ, se utilizaron para el análisis de valoración económica ambiental, según la metodología de Betanzos-Vega et al. (2014).

\section{Variantes productivas e instalaciones}

De acuerdo con los registros de la empresa pesquera (PESCATUN), la actividad ostrícola de Puerto Padre muestra dos variantes productivas: la recolecta de ostión silvestre y la cosecha del cultivo. La recolecta en bancos naturales es una actividad de pesca extractiva dependiente de existencias silvestres; el cultivo artesanal es una actividad tecnológica y productiva basada en la fijación de larvas del medio natural en sustratos colectores y su manejo posterior hasta la cosecha (Mazón-Suástegui et al. 2017).

La granja ostrícola de Puerto Padre tiene un diseño cubano tradicional (Nikolic et al. 1976) integrado por varias unidades de engorde, con líneas paralelas de 11 pares de postes de madera de mangle enterrados por un extremo en el fondo marino y sobresaliendo por encima del mar por el otro, formando una cama o meseta de donde se cuelgan 100 colectores. Los postes forman una estructura (empilotado) que se une por encima con varas de mangle que se utilizan como soporte para colgar los colectores, cada parque ostrícola cuenta con 10 unidades, 110 postes y 1000 colectores, distribuidos en un área de $250 \mathrm{~m}^{2}$, y una granja cuenta con uno o varios parques ostrícolas. Según el establecimiento pesquero de Puerto Padre, toda la madera utilizada en la granja, y los colectores de ostión, provienen del bosque de manglar que ha sido objeto de uso tradicional. En 2017, se amplió el número de unidades de engorde en la granja, para instalar unos 16 mil colectores, y en 2018 y 2019 se ampliaron las unidades de engorde para recibir 30000 y 33000 colectores respectivamente; todo lo cual condujo al uso de materia prima de manglar.

\section{Aplicación del cultivo ecosistémico}

En 2019 se trazó una estrategia para eliminar o reducir el uso de materia prima proveniente del manglar, como beneficio a la resiliencia de este ecosistema. Se aplicaron las siguientes acciones ecoamigables y medidas tendientes a la conservación de los BSA: (1) Para erradicar la tala de arbustos de mangle se compró madera para la confección de pilotes y varas para la reconstrucción de la granja; (2) en colaboración con la Empresa Agroforestal del mu- 
nicipio Puerto Padre, y de la Empresa Nacional para la Protección de la Flora y la Fauna (ENPFF), se trazó una estrategia para la recuperación de manglares afectados por la desforestación y por la actividad ostrícola y (3), por contrato con la ENPFF, se autorizó la poda responsable de ramas terminales aéreas de mangle rojo, siguiendo el procedimiento establecido para su uso como colector de ostión (Frías y Rodríguez 1991, Betanzos-Vega et al. 2014).

Para aplicar buenas prácticas de cultivo artesanal, la granja ostrícola se reubicó alejada de los bancos naturales en el manglar para disminuir el impacto al ecosistema y evitar fijaciones secuénciales de ostrillas en los colectores, para garantizar mayor homogeneidad de tallas en la cosecha (Mazón-Suéstegui et al. 2017). La recolecta de ostión silvestre se limitó a una talla mayor de $40 \mathrm{~mm}$ de longitud antero-posterior, talla comercial con un rendimiento promedio en carne mayor o igual al $5 \%$ (Betanzos-Vega et al. 2018).

\section{Relación costo-beneficio de la actividad ostrícola}

Se analizaron los costos por ampliación de la granja ostrícola (2017 - 2019), y se incluyeron datos de 2015 y 2016, los cuales según criterios empresariales son representativos de un periodo con predominio de la variante pesquera extractiva. No se incluyeron los costos por embarcación, fuerza laboral, gastos de combustibles y otros insumos logísticos, considerando su existencia desde antes de 2015, que son relativamente fijos para esta actividad independientemente de la variante de producción (pesca o cultivo).

En la valoración económica se tuvieron en cuenta los costos de inversión anual para el funcionamiento y ampliación de la granja, y los ingresos anuales por venta de la producción total de ostión desconchado. Según listado de precios de la empresa pesquera (PESCATUN), hasta abril de 2018 el ostión se comercializó a un precio equivalente a US\$ $1.50 \mathrm{~kg}^{-1}$ de carne de ostión en salmuera, pero a partir de mayo de 2018 la oficina de finanzas y precios del Órgano Local de Gobierno en Las Tunas, autorizó un precio en moneda nacional (CUP) que es equivalente US\$2.30 $\mathrm{kg}^{-1}$ lo que influyó en el análi- sis de costo-beneficio.

\section{Costos de inversión}

Se incluyó la adquisición anual de materiales indispensables para el funcionamiento y desarrollo de la ostricultura artesanal. En los costos de 2019, se incluyó el costo de 200 colectores fabricados con recortes de alambre de aluminio (Figura 2B) y 100 colectores tipo sarta confeccionados con concha madre de ostión (Figura 2C). Con estos nuevos colectores se sustituyó igual cantidad de colectores de ramas de mangle (Figura 2A).

El costo de confección e instalación de un colector, sin incluir el costo del cordel para su confección o amarre, varía según su tipo, y se expresó en USD según tasa de cambio oficial del dólar respecto a la moneda nacional ( 1 USD $=25$ CUP). Para la confección del colector de concha madre de ostión se utilizaron 30 conchas (valvas) por ristra y cuatro ristras por colector, con un costo total por manufactura (mano de obra) equivalente a US\$ 0.30 por colector. El costo fijado por la empresa por manufactura de colector de ramas de mangle es equivalente a US\$ 0.75 por cada 120 colectores. Los 200 colectores de alambre de aluminio se confeccionaron con recortes desechados de otras actividades industriales, el costo por manufactura fue de US\$ 0.15 por colector. EI alambre de aluminio utilizado, calibre \# 2/0 (3 mm de diámetro), se comercializa en rollos o trenzados de 6 filamentos con un alto costo, que se compensa con la durabilidad (Zayas y Frías 1989, Frías y Rodríguez 1991). Para amarrar los colectores a las empalizadas de la granja se utiliza nylon monofilamento (diámetro $3 \mathrm{~mm}$ ). El costo unitario de la madeja de $1000 \mathrm{~m}$ de nylon monofilamento es de US\$ 80.00; se utilizan $1.5 \mathrm{~m}$ para sostener cada colector de las varas o tendales, y $4.0 \mathrm{~m}$ de cordel para la confección del colector de conchas.

Para la valoración netamente económica, se calculó la ganancia bruta (GB) anual, resultado de la diferencia entre costos de inversión e ingresos económicos tangibles (por venta de ostión). Se obtuvo la ganancia neta (GN = rentabilidad económica) de la diferencia entre la ganancia bruta (GB) e ingresos por venta (IV) de ostión después de impuestos 
(35\%), sobre los ingresos: $G N=G B-\left(0,35^{*} \mathrm{IV}\right)$. Este es el gravamen impositivo sobre ventas establecido para las empresas estatales autofinanciadas (Betanzos-Vega et al. 2014).

\section{Valoración ambiental}

Se refiere al valor intangible de los BSA, el daño ambiental se reflejó en costos, considerando que un daño que se evita se refleja como un beneficio (ingreso), mientras que un beneficio ambiental que se pierde por daño al manglar se refleja como un costo (Gómez-País 2007). El costo ambiental por daños al ecosistema de manglar, se calculó de acuerdo al valor ecológico estimado por Gómez-País (2007), de Beneficio Bruto (mínimo = US\$500 ha ${ }^{-1}$ año $^{-1}$, medio $=$ US $\$ 1000 \mathrm{ha}^{-1} \mathrm{año}^{-1}$, y máximo $=$ US $\$$ $1500 \mathrm{ha}^{-1}$ año $^{-1}$ ), derivado de los servicios y funciones ecosistémicas del manglar.

Con base en estos criterios, se consideró que: 1) la tala total de arbustos de mangle para obtener pilotes y varas para la granja de cultivo, ejerce una máxima afectación al manglar y reduce sus funciones y oferta de BSA, lo cual se valoró en US $\$ 1500 \mathrm{ha}^{-1}$; 2) el corte o descortezado de las raíces de mangle para extraer el ostión silvestre se valoró en US\$ 1000 $\mathrm{ha}^{-1}$ al considerar un daño limitado a las funciones del manglar y servicios ecológicos de sus raíces, y 3 ) el corte o poda de las ramas terminales de mangle para la confección de colectores se estimó en US\$ $500 \mathrm{ha}^{-1}$, debido a un menor impacto sobre las funciones del manglar (Betanzos-Vega et al. 2014). Con razonamiento similar, se definieron los ingresos por beneficio ambiental, en función del valor derivado de los BSA preservados por reducción del daño al manglar o por sustituir el uso de sus componentes por otros de diferente origen, calculados en razón del beneficio bruto máximo, medio y mínimo indicados por Gómez-País (2007).

El ahorro por sustitución de materiales del manglar por otros recuperados, o de menor costo y similar utilidad, se consideró un ingreso debido a su beneficio económico. Durante 2018 y 2019 fue sustituido el $100 \%$ del monofilamento de nylon por cordel recubierto de caucho vulcanizado de $2.6 \mathrm{~mm}$ de diámetro, de alta resistencia y durabilidad recu- perado de la fibra textil de neumáticos desechados de camión y de tractor.

En el análisis de costo-beneficio global, valoración económica y ambiental, la ganancia bruta de cada año evaluado (2015 - 2019), se dedujo a partir de la diferencia entre los ingresos totales (ingresos por venta de ostión + ingresos por beneficio ambiental + ahorro de materiales) y los costos totales (costos de inversión + costo ambiental). Con este método, la ganancia bruta permite estimar los resultados económicos de la producción y el desempeño ambiental de la entidad productiva, mientras que la ganancia neta (rentabilidad económico - ambiental), es un indicador de viabilidad económica y factibilidad ambiental de la actividad, resultado de la valoración económica de los BSA del manglar.

\section{Análisis estadístico}

Los análisis estadísticos se realizaron con el programa STATGRAPHICS ${ }^{\circledR}$ Centurion XV (Statgraphics.Net, Madrid, España), de acuerdo con Zar (2019). Se realizaron pruebas de normalidad de datos (Prueba-F y verificación de la varianza para comparar las desviaciones estándar). Debido a normalidad de los datos, en el análisis comparativo entre la producción de ostión por pesca y cultivo se aplicó la prueba paramétrica de comparación de medias de $t$-Student. En la comparación de la abundancia $(\mathrm{kg})$ de ostión asociada a los tres tipos de colector, debido a que los datos mostraron atipicidad, se utilizó la prueba no paramétrica de Kruskal-Wallis, con salida gráfica de caja y bigotes. En todas las pruebas se utilizó un valor de significación $P=0,05$. Los valores promedio en el texto se expresan con la desviación estándar (media $\pm \mathrm{DE}$ ).

\section{RESULTADOS}

\section{La producción ostrícola en Puerto Padre}

La producción de ostión (2010-2019), reflejó una tendencia decreciente (Figura 3), con mínimos en 2016 (26.8 t) y 2017 (20.3 t), y recuperación a partir de 2018 por ampliación de la ostricultura. En el análisis comparativo (prueba-t) de la producción 


\section{Producción de ostión, Puerto Padre (Las Tunas)}

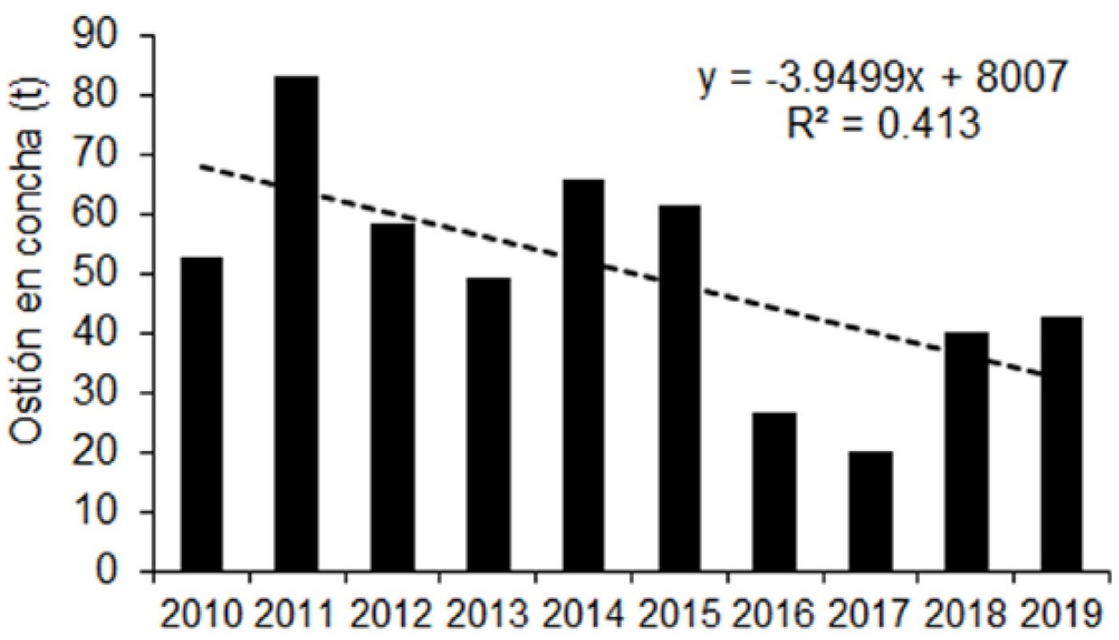

Figura 3. Variaciones en la producción total anual de ostión de mangle y tendencia lineal durante el periodo $2010-2019$.

anual (2015-2019), entre ambas variantes productivas, pesca y cultivo, no se detectó diferencia significativa ( $\mathrm{t}=-0.131916, \mathrm{P}=0.8983$ ) para un nivel de confianza del $95 \%$. La producción por cultivo mostró $18.48 \pm 12.17$ t y $19.86 \pm 19.98$ t la producción por pesca extractiva de ostión silvestre. En contraparte, a partir de 2017 predominó la producción por ostricultura (Figura 4). En términos generales, la producción anual por pesca o recolecta de ostión de los bancos naturales se redujo, mientras que la producción por ostricultura se incrementó de $7 \mathrm{t}$ durante 2015-2016, a $36.5 \mathrm{t}$ en 2019. El rendimiento porcentual promedio en carne (R) de 2015 al 2019 fue del 6.4\% para el ostión cultivado, mientras que para el ostión silvestre recolectado de los bancos naturales fue del $5.8 \%$, y el máximo promedio anual de rendimiento en carne de ostión (7.6\%) se alcanzó en 2019 , en ostión de cultivo (Tabla 1).

En 2015 el 83.4\% de la producción de ostión en concha provino de la pesca de ostión silvestre, y en 2019 el $84.1 \%$ de la producción total de ostión procedió de la ostricultura, lo que favoreció un incremento de $1.3 \%$ el rendimiento en carne $(R)$ en 2019, respecto al obtenido en 2015. En 2019, entre los diferentes tipos de colectores, se registró diferencia estadísticamente significativa (Kruskal-
Wallis $=47.84, P=4.0842$ ) en el rendimiento (kilogramos de ostiones por colector) para un nivel de confianza del $95 \%$. Con el colector de alambre de aluminio se obtuvo el mayor rendimiento promedio, siendo menor en el de ramas de mangle (Figura 5). De 32700 colectores de ramas de mangle se obtuvieron $35316 \mathrm{~kg}$ de ostión para una productividad o rendimiento promedio por colector de $1.08 \pm 1.22$ $\mathrm{kg}$ de ostión en concha. De los 200 colectores de alambre de aluminio, se cosecharon $780 \mathrm{~kg}$ de ostiones con rendimiento promedio de $3.9 \pm 0.98 \mathrm{~kg}$, y $300 \mathrm{~kg}$ en los 100 colectores de concha madre, con rendimiento promedio de $3 \pm 1.05 \mathrm{~kg}$ de ostión por colector. La abundancia media $(\mathrm{kg})$ por metro cuadrado en bancos naturales de ostión se estimó en $0.88 \pm 2.06 \mathrm{~kg}$.

\section{Costo-beneficio económico y ambiental de la ac- tividad ostrícola}

En la valoración netamente económica (Tabla 2), la rentabilidad fue positiva en cuatro de los cinco años evaluados (2015-2019). Aunque la mayor inversión por ostricultura ocurrió en 2018, solo en 2017 la rentabilidad económica fue negativa debido a incremento de los costos en ostricultura. A partir de 2018, se registraron incrementos en los precios de venta 


\section{Producción de ostión (Pesca y Cultivo)}

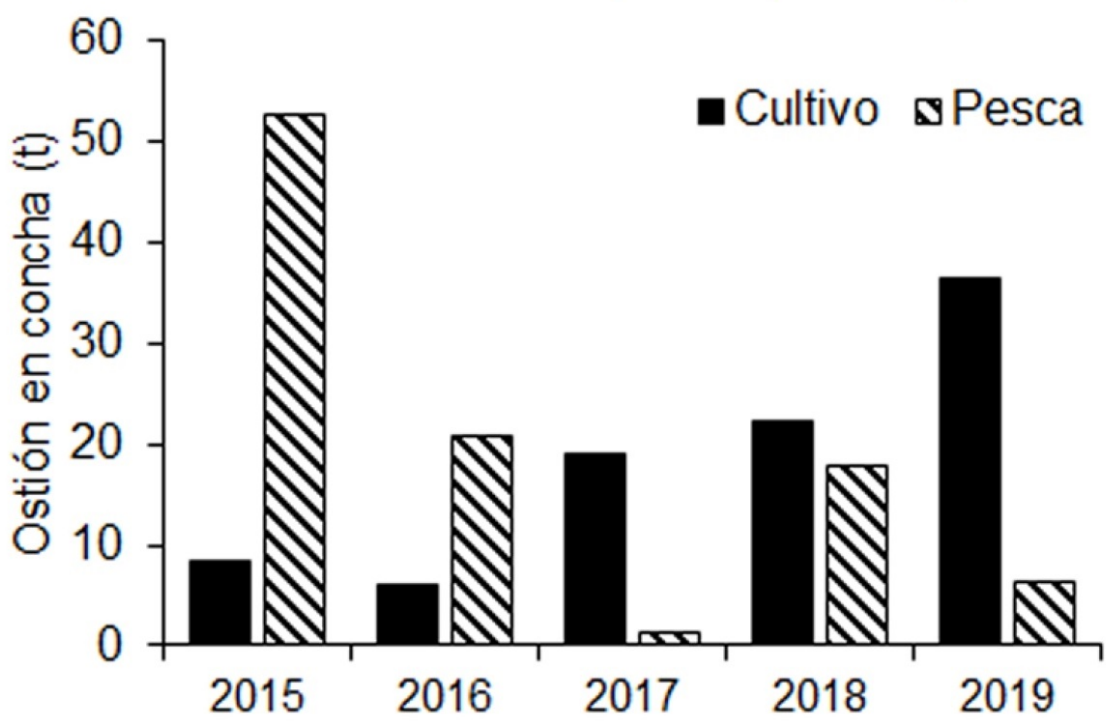

Figura 4. Variación anual de la producción de ostión en la bahía de Puerto Padre, Cuba (2015 2019), según resultados de la pesca extractiva del recurso silvestre y la cosecha a partir del cultivo artesanal.

Tabla 1. Producción ( $t$ ) y rendimiento en carne $(R \%)$ de ostión cosechado en granja (Cultivo) y recolectado en bancos naturales (Pesca), en Puerto Padre, Las Tunas, Cuba (2015-2019).

\begin{tabular}{lcccccc}
\hline & Cultivo $(\mathrm{t})$ & Cultivo $(\mathrm{R} \%)$ & Pesca $(\mathrm{t})$ & Pesca $(\mathrm{R} \%)$ & Total $(\mathrm{t})$ & Total $(\mathrm{R} \%)$ \\
\hline 2015 & 8.50 & 6.00 & 52.70 & 5.40 & 61.20 & 5.70 \\
2016 & 6.10 & 6.20 & 20.70 & 5.50 & 26.80 & 5.90 \\
2017 & 19.00 & 6.30 & 1.30 & 5.90 & 20.30 & 6.10 \\
2018 & 22.30 & 6.10 & 17.70 & 5.70 & 40.00 & 5.90 \\
2019 & 36.50 & 7.60 & 6.90 & 6.30 & 43.40 & 7.00 \\
Promedio & 18.50 & 6.40 & 19.90 & 5.80 & 38.30 & 6.10 \\
Desviación estándar $( \pm)$ & 12.20 & 0.60 & 19.80 & 0.36 & 15.90 & 0.51 \\
\hline
\end{tabular}

de ostión. El efecto del precio de venta también se notó en los ingresos de 2015, ya que a pesar de una máxima producción (61.2 t), la rentabilidad fue menor ese año que la de 2019 con producción de 43.8 t. Otro factor que redujo costos en 2018 y 2019 , fue la sustitución del cordel de nylon monofilamento por el cordel de llantas desechadas, lo que evitó gastos significativos (Tabla 2).

La inclusión de los costos ambientales (valoración ambiental), en el análisis económico de costobeneficio, influyó en el incremento de los costos totales (valoración económico-ambiental), debido al impacto a los BSA del ecosistema, sobre todo en 2015 y 2016 cuando predominó la actividad por pesca extractiva de ostión silvestre, y en 2018 y 2019 por incremento en el uso de ramas de mangle para la confección de colectores. Esta situación se reflejó en una rentabilidad económico - ambiental negativa de 2015 a 2017 (Tabla 2). Pero a partir del desarrollo de la ostricultura desde 2018, y de un mayor número de acciones eco-amigables en 2019 , fue posible revertir progresivamente la afectación a los BSA que presta el manglar, lo que tuvo consecuencia positiva en la rentabilidad económica ambiental a partir de 2018.

\section{DISCUSIÓN}

\section{La producción ostrícola en Puerto Padre}

Las medidas eco-amigables y otras acciones promovidas para el desarrollo del cultivo artesanal 


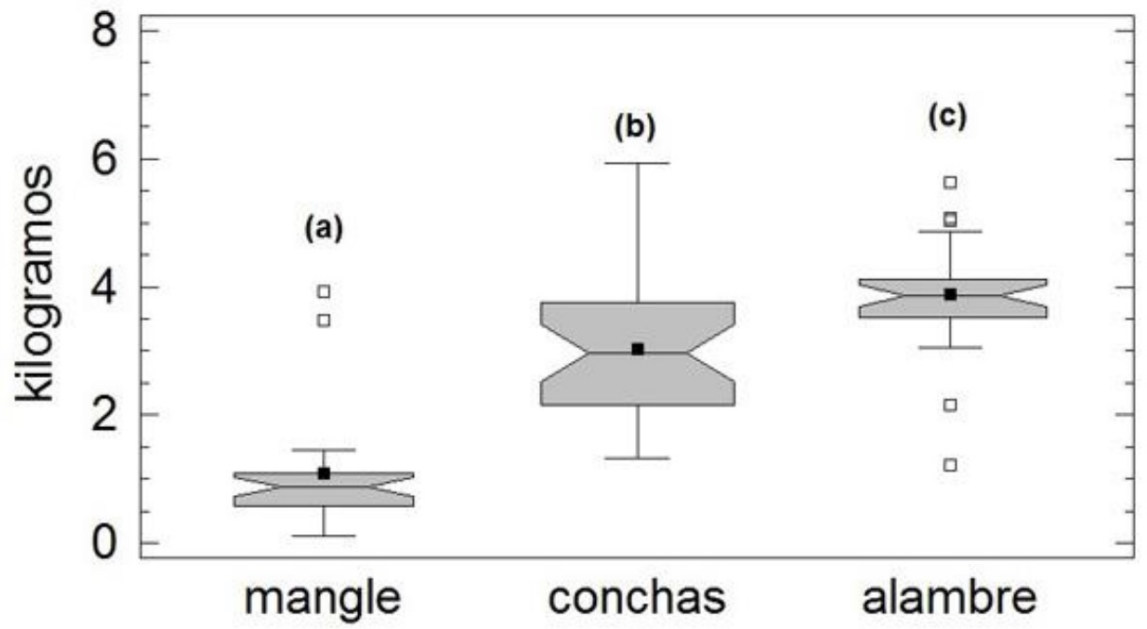

Figura 5. Comparación (Kruskal-Wallis) entre tipos de colectores de ostión: ramas de mangle, sarta de concha madre, y alambre de aluminio. Letras desiguales $(a, b, c)$ indican medianas significativamente diferentes, con probabilidad del $95 \%$.

Tabla 2. Análisis de costo - beneficio (\$USD) de la producción de ostión (2015 - 2019) en la bahía de Puerto Padre. Incluyendo valoración ambiental según costos por daños e ingresos por beneficio de BSA del manglar.

\begin{tabular}{|c|c|c|c|c|c|}
\hline Concepto / año & 2015 & 2016 & 2017 & 2018 & 2019 \\
\hline \multicolumn{6}{|l|}{ Valoración económica } \\
\hline \multicolumn{6}{|l|}{ Costos económicos (inversión): } \\
\hline -Compra de madera para granja & 0.00 & 0.00 & 0.00 & 2780.00 & 1150.00 \\
\hline -Cordel de nylon para colectores & 960.00 & 720.00 & 2000.00 & 0.00 & 0.00 \\
\hline -Puntillas (clavos de 5 pulgadas) & 75.00 & 10.00 & 85.00 & 175.00 & 50.00 \\
\hline -Colectores de ramas de mangle & 43.75 & 31.25 & 112.50 & 156.25 & 206.25 \\
\hline -Colectores de concha madre & 0.00 & 0.00 & 0.00 & 0.00 & 56.00 \\
\hline -Colectores de alambre aluminio & 0.00 & 0.00 & 0.00 & 0.00 & 10.00 \\
\hline Costos de inversión & 1078.75 & 761.25 & 2197.50 & 3111.25 & 1472.25 \\
\hline \multicolumn{6}{|l|}{ Ingresos económicos: } \\
\hline - Ingresos por venta de ostión & 5108.40 & 2310.90 & 1786.00 & 5441.00 & 6892.00 \\
\hline Ganancia bruta & 4029.65 & 1549.65 & -411.50 & 2329.75 & 5419.75 \\
\hline Rentabilidad económica & 2241.71 & 740.84 & -1036.60 & 425.40 & 3007.55 \\
\hline \multicolumn{6}{|l|}{ Valoración ambiental } \\
\hline \multicolumn{6}{|l|}{ Daño ambiental (pérdida de BSA): } \\
\hline -Pesca extractiva & 7905.00 & 3105.00 & 195.00 & 2655.00 & 1035.00 \\
\hline -Tala de mangles & 210.00 & 180.00 & 285.00 & 120.00 & 0.00 \\
\hline - Corte de ramas para colectores & 450.00 & 325.00 & 975.00 & 1.375 .00 & 1.650 .00 \\
\hline Costo ambiental & 8565.00 & 3610.00 & 1455.00 & 4150.00 & 2685.00 \\
\hline \multicolumn{6}{|l|}{ Beneficio ambiental: } \\
\hline -Reducción de pesca extractiva & 425.00 & 305.00 & 950.00 & 1115.00 & 1604.00 \\
\hline -Supresión de la tala de mangle & 0.00 & 0.00 & 0.00 & 0.780 & 1150.00 \\
\hline -Reducción del corte de ramas & 0.00 & 0.00 & 0.00 & 0.00 & 54.00 \\
\hline Ingresos por beneficio ambiental & 425.00 & 305.00 & 950.00 & 3895.00 & 2808.00 \\
\hline \multicolumn{6}{|l|}{ Beneficio por ahorro de materiales: } \\
\hline •Sustitución de cordel de nailon & 0.00 & 0.00 & 0.00 & 2.480 .00 & 400.00 \\
\hline \multicolumn{6}{|l|}{ Valoración económica Ambiental } \\
\hline Costo total (económico-ambiental) & 9643.75 & 4371.25 & 3652.5 & 7261.25 & 4157.25 \\
\hline Ingreso total (económico-ambiental) & 5533.40 & 2615.90 & 2736.00 & 11816.00 & 10100.00 \\
\hline Ganancia Bruta & -4110.35 & -1755.35 & -916.50 & 4554.75 & 5942.75 \\
\hline Rentabilidad económica-ambiental & -5898.29 & -2564.17 & -1541.60 & 2650.40 & 3530.55 \\
\hline
\end{tabular}

ecosistémico en Puerto Padre, posterior a 2017, tuvieron resultados positivos en la productividad. El análisis del costo por inversiones para la ostricultura indica que la pesca extractiva de ostión es más 
rentable que el cultivo artesanal, pero diferentes criterios bioecológicos demuestran que la pesca extractiva de ostión silvestre no es selectiva con respecto a la talla, debido a la sobre-fijación de larvas sobre juveniles y adultos (Mazón-Suástegui et al. 2017). En los bancos naturales se incrementa la competencia por espacio, alimento y oxígeno, y se registra un menor índice de condición del ostión (Betanzos-Vega et al. 2014, Mazón-Suástegui et al. 2017, BetanzosVega et al. 2018), y esto se confirmó con un mayor rendimiento en carne $(R)$ obtenido en ostión de cultivo que, en ostión silvestre, lo que demuestra que solo el cultivo puede incrementar de manera sostenible la producción ostrícola (Mazón-Suástegui et al. 2019).

La mayor productividad en kilogramos de ostión por colector se obtuvo en las unidades de alambre de aluminio y de concha madre tipo sarta, versus el colector tradicional de ramas de mangle. Esto contradice el criterio popular entre los ostricultores cubanos, de que es mayor la tasa de fijación de ostión en el colector de ramas de mangle, porque las larvas de OM se fijan de manera preferente en el mangle rojo, su principal sustrato natural.

\section{Costo-beneficio económico y ambiental de la ac- tividad ostrícola}

Al incluir la valoración ambiental en el análisis de costo-beneficio de la actividad ostrícola en Puerto Padre, se redujo el impacto al manglar en 2018 y 2019, y la actividad fue más rentable. El análisis de valoración económica ambiental, demostró que la introducción del cultivo con enfoque ecosistémico generó mayor beneficio ambiental e incrementó la productividad, lo que coincide con otros estudios de flujo de caja y factibilidad económico-ambiental de la ostricultura ecosistémica, respecto a la pesca extractiva combinada con la cría y engorde de ostión en colectores de mangle suspendidos del propio manglar (Betanzos-Vega et al. 2014). Independientemente del incremento en el precio de venta del ostión desde mediados de 2018, y del enfoque precautorio en la captura de ostión silvestre, la aplicación de buenas prácticas en el cultivo permitió un aumento en la producción y en el rendimiento en carne de ostión del 7.0\% en 2019. Estos resultados, refuerzan la hipóte- sis de que el cultivo es, a la postre, la opción más sustentable frente a la recolecta de ostión silvestre (Mazón-Suástegui et al. 2019, Betanzos-Vega et al. 2020), que indica que la actividad extractiva puede ser económicamente viable en el corto plazo, pero no ambientalmente factible en el mediano y largo plazo, por el daño que recibe el ecosistema. A las presiones de la pesca se suman la vulnerabilidad del ostión y del manglar ante los efectos del cambio climático, y la antropización de la zona costera (FAO 2011, DíazAbreu et al. 2018), lo que incide en la reducción de la biomasa silvestre y en una menor producción a largo plazo. La pérdida de BSA del manglar por degradación de ecosistemas no es entendida como un factor colateral en la reducción de la productividad de actividades que hacen un uso no sostenible de ellos (Barsev 2008, Díaz-Abreu et al. 2018). Por lo anterior, es necesario incrementar acciones para preservar o recuperar dichos BSA, replicando estas experiencias de adaptación pesquera basada en la protección de los ecosistemas, en la gestión de los recursos naturales de Cuba, y en otros países o regiones.

\section{CONCLUSIONES}

El cultivo artesanal de ostión con enfoque ecosistémico es una alternativa a la pesca del recurso silvestre, y de mayor rendimiento productivo. Se demostró la viabilidad de aplicar medidas ecoamigables para preservar los BSA del manglar y reducir las presiones de la actividad pesquera extractiva. Es factible el uso de colectores artificiales y de concha madre de ostión, para incrementar la producción, y reducir el impacto al ecosistema sustituyendo los colectores confeccionados con ramas de mangle. La cuantificación económica de la oferta de BSA del manglar, así como la inclusión del valor ecológico por daños y beneficios al ecosistema de manglar en el análisis de costo-beneficio de la actividad ostrícola demostró la factibilidad del cultivo artesanal de ostión con enfoque ecosistémico, y su potencialidad a futuro, para una certificación ecológica de esta pesquería. 


\section{AGRADECIMIENTOS}

Al proyecto GEF / LDCF / SCCF "Incorporando consideraciones ambientales múltiples y sus implicaciones económicas en el manejo de paisajes, bosques y sectores productivos en
Cuba"(ECOVALOR). Al fondo Sectorial de Investigación para la Educación (México), proyectos CBConacyt 258282 y PROINNOVA-Conacyt 241777, desarrollados en CIBNOR, S.C. A los programas académicos EDI y COFFA, de CICIMAR-IPN.

\section{LITERATURA CITADA}

ACC (1989) Nuevo Atlas Nacional de Cuba. Instituto de Geografía de la Academia de Ciencias de Cuba, Instituto de Geodesia y Cartografía, e Instituto Geográfico Nacional de España. Madrid, España. 205p.

Alcolado PM, García EE, Arellano-Acosta M (2007) Ecosistema Sabana-Camagüey. Estado actual, avances y desafíos en la protección y uso sostenible de la biodiversidad. Proyecto PNUD/GEF Sabana-Camagüey CUB/98/G32; CUB/99/G81. Editorial Academia. Cuba. 183p.

Barsev R (2008) Mecanismos financieros para la conservación de recursos naturales: guía metodológica. Editorial Academia, La Habana, Cuba. 95p.

Betanzos-Vega A, Rivero-Suárez S, Mazón-Suástegui JM (2014) Factibilidad económico-ambiental para el cultivo sostenible de ostión de mangle Crassostrea rhizophorae (Guilding 1828) en Cuba. Latin American Journal of Aquatic Research 42: 1148-1158.

Betanzos-Vega A, Capetillo-Piñar N, Latisnere-Barragán H, Ortiz-Cornejo NL, Mazón-Suástegui JM (2018) Oyster production and meat yield in Crassostrea spp (bivalvia: Ostreidae) in Pinar del Rio, Cuba. Revista Ecosistemas y Recursos Agropecuarios 5: 501-510.

Betanzos-Vega A, Mazón-Suástegui JM, Tripp-Quesada A, Jaime-Ceballos B, Arencibia-Carballo G, LeyvaSegura AR (2020) Potencial ostrícola de Crassostrea rhizophorae (Guilding, 1828), en las bahías de Manatí y Puerto Padre, provincia Las Tunas, Cuba. Revista Cubana de Investigaciones Pesqueras 37: 71-76.

Díaz-Abreu DM, González-Mejías Y, Pérez-Fernández R (2018) Bienes y servicios ecosistémicos. Estudio de caso: Valoración económica de reserva ecológica bahía Nuevas Grandes - La Isleta. Las Tunas. Cuba. Revista Digital de Medio Ambiente 52: 1-26.

FAO (2011) Desarrollo de la acuicultura. Enfoque ecosistémico a la acuicultura. Orientaciones Técnicas para la Pesca Responsable. No. 5, Supl. 4. Roma, FAO. 60p.

Frías JA, Rodríguez J (1991) Oyster culture in Cuba: Current state, techniques and industry organization. In: Newkirk GF, Field BA (eds). Oyster culture in the Caribbean. Proceeding of a Workshop. Kingston, Jamaica. Mollusc Culture Network. Halifax, Canada. pp: 51-74.

Gómez-País G (2007) Análisis económico de las funciones ambientales del manglar en el ecosistema SabanaCamagüey. En: Alcolado PM, García E, Arellanos M (eds). Ecosistema Sabana-Camagüey. Estado actual, avances y desafíos en la protección y uso sostenible de la biodiversidad. Proyecto PNUD/GEF SabanaCamagüey CUB/98/G32 y CUB/99/81. La Habana, Cuba. pp: 159-164.

López S (2019) Desplazamiento forzado y degradación del mangle Tumaco: una visión desde la valoración integral (VBSE). Boletín Semillas ambientales 13: 68-74.

Mazón-Suástegui JM, Rivero-Suárez SE, Betanzos-Vega A, Saucedo PE, Rodríguez-Jaramillo C, Acosta-Salmón $\mathrm{H}$ (2017) Potential of sites in northern Cuba for developing an industry of the native mangrove oyster (Crassostrea rhizophorae). Latin American Journal of Aquatic Research 45: 218-222. 
Mazón-Suástegui, JM, Tripp-Quezada A, Betanzos-Vega A (2019) Diagnóstico de la producción de ostión (Bivalvia: Ostreidae) en Cuba, ventajas de Crassostrea virginica (Gmelin. 1791) para la ostricultura. Revista de Investigaciones Marinas 39: 105-118.

Nikolic M, Bosch AC, Alfonso SJ (1976) A system for farming the mangrove oyster Crassostrea rhizophorae. Guilding (1828). Aquaculture 9: 1-18.

Palacios ML, Cantera JR (2017) Mangrove timber use as an ecosystem service in the Colombian Pacifi. Hydrobiology 803: 345-35.

Rebelo MF, Amaral MCR, Pfeifer WC (2005) Oyster condition index in Crassostrea rhizophorae (Guilding, 1828) from a heavy-metal polluted coastal lagoon. Brazilian Journal of Biology 2: 345-351.

Zayas CR, Frías JA (1989) Estudio comparativo entre colectores de alambre y de mangle en el cultivo comercial de ostión (Crassostrea rhizophorae) en Cuba. Revista de Investigaciones Marinas 10: 125-132.

Zar JH (2019) Biostatistical analysis. 5th Edition. Prentice Hall. New Jersey. 66p. 